\title{
Características Clínicas, Diagnósticas e Laboratoriais de Portadores de Carcinoma Bronquioloalveolar
}

\author{
Clinical, Diagnostic, and Laboratory Characteristics of Patients with \\ Bronchoalveolar Carcinoma
}

Sérgio Jamnik, Illka Lopes Santoro, Eliana Lourenço Borges, Wilner Vieira da Silva, César Uehara

\section{Resumo}

O carcinoma bronquioloalveolar (CBA) é um subtipo de adenocarcinoma, representa menos de 5\% dos tumores primários do pulmão, e possui características patológicas próprias. Poucos trabalhos foram publicados no Brasil. Objetivo: Estudar as características do carcinoma bronquioloalveolar em pacientes com diagnóstico de carcinoma broncogênico. Métodos: Estudo retrospectivo com levantamento de dados dos prontuários de pacientes do ambulatório de Oncopneumologia da UNIFESP (São Paulo). Resultados: Dos 1.617 pacientes com diagnóstico de carcinoma broncogênico, 45 (2,8\%) eram CBA. Predominou o sexo feminino (53,3\%), a cor branca (>70\%) e 64,4\% dos pacientes apresentaram história de tabagismo. Os principais sintomas foram: tosse (80\%), expectoração (68,9\%) e dispnéia (60\%). Os pacientes levaram em média 10,27 meses para procurar o ambulatório. As imagens radiológicas predominantes foram de padrão difuso. O método diagnóstico mais utilizado foi a broncoscopia $(71,2 \%)$. Conclusão: Os resultados encontrados são semelhantes aos da literatura mundial. No Serviço de Oncopneumologia da UNIFESP, se faz mais diagnósticos pela biópsia por broncoscopia.

Palavras-chave: Câncer de pulmão, Carcinoma bronquioloalveolar, Características clínicas 


\section{INTRODUÇÃO}

O câncer de pulmão é um dos cânceres de maior incidência, sendo a principal causa de morte relacionada ao câncer no mundo e no Brasil. A sua incidência no mundo é de aproximadamente 1,2 milhão/ano e sua mortalidade de 1 milhão/ano de pacientes ${ }^{1}$. No Brasil, a sua incidência é de mais de 25 mil pacientes/ano, sendo uma das principais causas de morte entre os cânceres².

$\mathrm{O}$ carcinoma de células não-pequenas, incluindo o carcinoma espinocelular, o adenocarcinoma e o carcinoma de células grandes, representa $80 \%$ a $85 \%$ de todos os cânceres de pulmão. A cirurgia neste tipo de câncer é o único tratamento que pode efetivamente levar à cura, mas somente em $15 \%$ a $20 \%$ dos pacientes consegue-se ressecção radical, com uma sobrevida de $40 \%$, em 5 anos. Desde que a maior parte desses doentes tenha doença avançada no diagnóstico, o principal tratamento é a quimioterapia ${ }^{3}$.

O CBA é um subtipo do adenocarcinoma, com características e patologia próprias, representando de $2 \%$ a $3 \%$ dos tumores primários de pulmão, cuja incidência tem aumentado nos últimos anos ${ }^{4}$.

O CBA é uma neoplasia bem diferenciada, que surge em brônquio periférico, estendendo-se para o espaço aéreo distal, preservando e usando a estrutura do pulmão como estroma (crescimento lepídico). Apresenta crescimento lento e, geralmente, tem bom prognóstico ${ }^{4,5}$. Este tipo histológico associa-se às seguintes características: mulheres, não-fumantes, orientais e apresenta melhor resposta à quimioterapia biológica, como genfitinibe, erlotinibe e anticorpos monoclonais dirigidos contra os receptores do fator de crescimento epitelial ${ }^{4}$. Isto provavelmente deve-se à mutação no domínio de tirosinaquinase, que causou um ganho de função desses receptores ${ }^{4}$. Poucos trabalhos foram publicados sobre este tipo histológico, sendo, portanto, as suas características pouco conhecidas no país. Este trabalho objetivou estudar as características de pacientes dessa neoplasia, para ampliar os conhecimentos sobre a epidemiologia, a clínica, o diagnóstico e os exames laboratoriais.

\section{METODOLOGIA}

Foram analisados os prontuários dos pacientes portadores de carcinoma bronquioloalveolar, de 1986 a 2005, atendidos no ambulatório de Oncopneumologia da Universidade Federal de São Paulo (UNIFESP). Todos os pacientes tiveram diagnóstico de carcinoma de pulmão comprovado por meio de exames anatomopatológicos. Foram avaliados dados referentes a: sexo, idade, cor, hábito tabágico, índice de performance status (Karnofsky), sinais, sintomas e tempo decorrido entre o início da sintomatologia e a primeira consulta no ambulatório (meses), imagem radiológica e/ou de tomografia computadorizada de tórax, principais métodos utilizados para o diagnóstico, exames laboratoriais, estadiamento da doença e terapia realizada. Para a avaliação de quantidade de cigarros fumados, utilizou-se o índice maços/ano.

As imagens radiológicas foram classificadas como focal e difusa, sendo que a primeira foi definida como acometendo até um lobo e, a segunda, mais de um lobo pulmonar. Em relação ao estadiamento, a doença foi definida como localizada e avançada. Na primeira, foram incluídos os estádios Ia, Ib, IIa, IIb e IIIa e, na segunda, os estádios IIIb e IV. Os exames laboratoriais séricos avaliados foram: velocidade de hemossedimentação (VHS), desidrogenase lática (DHL) e antígeno carcinoembrionário (CEA). Foram considerados valores normais, respectivamente, até $10 \mathrm{~mm}$, 480U/L e 10 nanogramas $/ \mathrm{ml}$.

$\mathrm{Na}$ análise estatística foram calculadas a média, a mediana e o desvio-padrão (DP) das variáveis contínuas. Em alguns casos, realizou-se o teste do qui-quadrado e o teste $t$ de Student. O nível de significância para rejeição da hipótese de nulidade foi sempre igual ou menor do que $0,05(5 \%)^{6}$. O trabalho foi submetido à Comissão de Ética da instituição.

\section{RESULTADOS}

No período estudado, o total de pacientes com diagnóstico de carcinoma broncogênico foi de 1.617. Destes, 45 (2,8\%) tiveram o diagnóstico de carcinoma bronquioloalveolar (CBA), sendo que $21(46,7 \%)$ pacientes eram do sexo masculino e $24(53,3 \%)$, do sexo feminino. Predominou no grupo a cor branca $(\mathrm{n}=32$ - 71,1\%), seguida de negros ( $\mathrm{n}=7-15,5 \%)$ e amarelos $(\mathrm{n}=6-13,3 \%)$. Não houve diferença significativa entre o grupo masculino e o grupo feminino, nem diferença em relação à média de idade entre os dois grupos, que foi em torno de 63 anos. O índice de Karnofsky era próximo em ambos os sexos ( $\mathrm{Md}=80,0)$ (Tabela 1$)$.

Os sinais e sintomas observados foram: tosse (80\%), expectoração $(68,9 \%)$ e dispnéia $(60 \%)$. As mulheres apresentaram uma tendência a ter mais tosse $(87,5 \% \mathrm{x}$ $71,4 \%)$, dispnéia $(75 \% \times 42,8 \%)$, perda ponderal $(58,3 \% \times 38,1 \%)$ e anorexia $(29,2 \%$ x $19,0 \%)$ (Tabela 2). No entanto, essas diferenças não foram significantes.

Os pacientes levaram, em média, 10,27 $\pm 10,54$ meses do início da sintomatologia até a primeira consulta no ambulatório. Não houve diferença entre os grupos masculino e feminino $(\mathrm{p}=0,63)$. Em cinco pacientes, não foi possível obter dados (3 homens e 2 mulheres) (Tabela 3). 
Tabela 1. Características da população estudada estratificada por sexo

\begin{tabular}{|l|l|l|l|l|}
\hline \multicolumn{1}{|c|}{ Características } & \multicolumn{1}{c|}{$\begin{array}{c}\text { Grupo } \\
\text { masculino }\end{array}$} & $\begin{array}{c}\text { Grupo } \\
\text { feminino }\end{array}$ & \multicolumn{1}{|c|}{ Total } & Valor de p \\
\hline Brancos (n - \%) & $15(71,4 \%)$ & $17(70,8 \%)$ & $32(71,1 \%)$ & $7(15,5 \%)$ \\
Negros (n- \%) & $3(14,4 \%)$ & $4(16,7 \%)$ & $6(13,3 \%)$ \\
Amarelos (n - \%) & $3(14,3 \%)$ & $3(12,5 \%)$ & $63 \pm 9,2$ & 0,52 \\
Idade (média) em anos & $62 \pm 8,9$ & $63,9 \pm 10,6$ & 64 & 0,59 \\
Idade (mediana) em anos & 62 & 65,5 & $29(64,4 \%)$ & \\
Tabagista (n - \%) & $17(80,9 \%)$ & $12(50 \%)$ & $37,2 \pm 21,05$ & \\
Maços/ano (média) & $41,5 \pm 23,16$ & $31,2 \pm 16,73$ & 39 & 0,48 \\
Maços/ano (mediana) & 41,0 & 37,2 & 80 & \\
Índice de Karnofsky (mediana) & 85 & 80 & \\
\hline
\end{tabular}

Tabela 2. Sinais e sintomas da população estudada estratificada por sexo

\begin{tabular}{l|l|l|l}
\hline \multicolumn{1}{c|}{ Sintomas } & Grupo masculino & Grupo feminino & Total \\
\hline Tosse & $15(71,4 \%)$ & $21(87,5 \%)$ & $36(80 \%)$ \\
Expectoração & $15(71,4 \%)$ & $16(66,7 \%)$ & $31(68,9 \%)$ \\
Dispnéia & $9(42,8 \%)$ & $18(75,0 \%)$ & $27(60 \%)$ \\
Perda ponderal & $8(38,1 \%)$ & $14(58,3 \%)$ & $22(48,9 \%)$ \\
Quadro neurológico & $8(38,1 \%)$ & $9(37,5 \%)$ & $17(39,4 \%)$ \\
Anorexia & $4(19,0 \%)$ & $7(29,2 \%)$ & $11(24,4 \%)$ \\
\hline
\end{tabular}

Tabela 3. Tempo decorrido entre o início da sintomatologia e a primeira consulta ambulatorial nos pacientes estudados

\begin{tabular}{l|c|c|c|c|c}
\hline & $\mathbf{n}$ & $\begin{array}{c}\text { Média } \\
\text { (meses) }\end{array}$ & $\begin{array}{c}\text { Mediana } \\
\text { (meses) }\end{array}$ & $\begin{array}{c}\text { Mínimo } \\
\text { (meses) }\end{array}$ & $\begin{array}{c}\text { Máximo } \\
\text { (meses) }\end{array}$ \\
\hline Total de pacientes & 40 & $10,27 \pm 10,54$ & 6,5 & 1 & 36 \\
Grupo masculino & 18 & $9,72 \pm 9,42$ & 6,5 & 1 & 36 \\
Grupo feminino & 22 & $10,72 \pm 11,59$ & 6,5 & 1 & 36 \\
\hline
\end{tabular}

$\mathrm{p}=0,631$

A maior parte dos exames radiológicos mostrou imagens focais $(53,3 \%)$. Estas foram em maior número no grupo masculino; no entanto, essa diferença não foi significante (Tabela 4).

A maioria $(71,1 \%)$ dos diagnósticos foi obtida por meio da broncoscopia, pela biópsia transbrônquica (60\%) e brônquica $(11,1 \%)$. Cerca de $26,7 \%$ dos pacientes obtiveram o seu diagnóstico através da cirurgia a céu aberto. Ainda se fez diagnóstico em $8,9 \%$ por punção pleural, $6,7 \%$ por lavado bronquioloalveolar e $4,4 \%$ por citológico de escarro.

Em cinco pacientes (3 homens, 2 mulheres), não houve informações suficientes para o estadiamento. Dos 40 pacientes restantes, $16(40 \%)$ foram classificados como doença localizada e $24(60 \%)$ como doença avançada. Não houve diferença entre os grupos masculino e feminino (Tabela 3).

Em relação ao tratamento, 16 pacientes $(35,6 \%)$ foram submetidos à cirurgia; 20 (44,4\%), à quimioterapia; e $6(13,3 \%)$, à radioterapia, associadas ou não a outro tratamento. Foram submetidos à cirurgia isoladamente 9 pacientes; à quimioterapia isoladamente 13; e à radioterapia isoladamente 2 pacientes.

O tratamento de suporte foi oferecido a $11(24,4 \%)$ pacientes. Dos pacientes submetidos à cirurgia, realizouse a lobectomia em $84 \%$.

Dos 14 pacientes que realizaram o exame de VHS, $12(85,7 \%)$ apresentaram valores acima do normal. Só realizaram os exames, respectivamente, de DHL e de CEA, 23 e 22 pacientes, com valores acima do normal em $4(17,4 \%)$ e em 7 (31,8\%), respectivamente. Os valores são mostrados na Tabela 5 . 
Tabela 4. Imagens radiológicas e estadiamento clínico encontrados na população estudada, estratificada por sexo

\begin{tabular}{|l|c|c|c|c}
\hline & $\begin{array}{c}\text { Grupo } \\
\text { masculino }\end{array}$ & $\begin{array}{c}\text { Grupo } \\
\text { feminino }\end{array}$ & Total & Valor de p \\
\hline Imagem & $14(56 \%)$ & $10(50 \%)$ & $24(53,3 \%)$ & \\
\hline $\begin{array}{l}\text { Focal } \\
\text { Difusa }\end{array}$ & $11(44 \%)$ & $10(50 \%)$ & $21(46,7 \%)$ & 0,76 \\
\hline Estadiamento & $7(38,9 \%)$ & $9(40,9 \%)$ & $16(40 \%)$ & \\
$\quad$ Doença localizada & $11(61,1 \%)$ & $13(59,1 \%)$ & $24(60 \%)$ & 1,0 \\
\hline Doença avançada &
\end{tabular}

Tabela 5. Resultado dos principais exames laboratoriais na população estudada

\begin{tabular}{|c|c|c|c|c|c|c|c|c|}
\hline \multirow{2}{*}{$\begin{array}{l}\text { Valores } \\
\text { Exames }\end{array}$} & \multicolumn{3}{|c|}{ Gerais } & \multicolumn{3}{|c|}{ Acima do normal } & \multirow{2}{*}{ Mínimo } & \multirow{2}{*}{ Máximo } \\
\hline & $\mathbf{n}$ & Média & Mediana & $\mathbf{n}$ & Média & Mediana & & \\
\hline VHS (mm) & 14 & $49,4 \pm 37,96$ & 37,5 & 12 & $57,8 \pm 20,87$ & 45 & 1 & 122 \\
\hline DHL (U/L) & 23 & $300,7 \pm 163,55$ & 248 & 4 & $588,2 \pm 74,11$ & 607 & 92 & 652 \\
\hline CEA $(\mathrm{ng} / \mathrm{ml})$ & 22 & $19,16 \pm 27,36$ & 23 & 7 & $54,78 \pm 20,87$ & 60 & 0,4 & 80 \\
\hline
\end{tabular}

Valores normais: VHS até $10 \mathrm{~mm}$; DHL até 480U/l; CEA até 10 nanograma/ml

\section{DISCUSSÃO}

Apesar de o carcinoma bronquioloalveolar ser considerado um subtipo do adenocarcinoma, ele apresenta diferenças clínicas e patológicas relevantes, o que justifica o seu estudo. Neste trabalho, ele apresentou 2,5\% dos carcinomas broncogênicos, próximo aos dados da literatura mundial que citam valores inferiores a $5 \%$. Observa-se na literatura que, nos últimos anos, este tipo histológico tem apresentado um aumento ${ }^{1,7,8}$. Nos Estados Unidos, antes de 1980, ele representava 5\% de todos os adenocarcinomas, passando para $20 \%$ a $24 \%$, na década de $1990^{4,5,9,10}$.

Como nas literaturas espanhola e norte-americana, no presente trabalho houve predomínio do sexo feminino e de pacientes não-fumantes (Tabela 1), com os valores da idade no momento do diagnóstico em torno de 60 anos $^{5,11}$. Os pacientes com CBA tendem a ser mais jovens do que aqueles com carcinomas de células não-pequenas e mais de $30 \%$ não são fumantes ${ }^{12,13}$.

A quantidade de maços/ano foi superior entre os homens $(41,5)$ em relação às mulheres $(31,2)$, mostrando, como nos outros tipos histológicos, que com menor quantidade de cigarros fumados elas desenvolvem o tumor ${ }^{3}$.

As manifestações clínicas foram pouco expressivas e inespecíficas, com o predomínio da tosse $(80 \%)$, expectoração (broncorréia) $(68,9 \%)$ e dispnéia $(60 \%)$, sendo a broncorréia o sinal mais típico. Esta e a perda de peso $(48,9 \%)$ são consideradas marcadores de doença avançada. A broncorréia ocorre porque parte do CBA origina-se de células colunares produtoras de muco (tipo mucinoso) e, por ser multicêntrico, tem pior prognóstico do que os não-mucinosos que se originam dos pneumócitos tipo 2 ou de células claras que são mais localizadas $^{5}$. No presente trabalho, observou-se, em ambos os sexos, um longo tempo entre o início das manifestações clínicas e a primeira consulta no ambulatório de oncopneumologia. Este retardo no atendimento e no diagnóstico da doença deve-se à inespecificidade dos sintomas e da radiografia, o que faz com que os médicos pensem em outras doenças pulmonares mais comuns, como Doença Pulmonar Obstrutiva Crônica (DPOC) e pneumonia, em até 60\% dos $\operatorname{casos}^{9,14-16}$.

Neste trabalho predominou a doença com imagem radiológica focal $(53,3 \%)$, semelhante ao estudo de Fernandéz et al. ${ }^{5}$ (57\%). Este achado deve-se, principalmente, ao seu crescimento lento, levando meses para a doença se disseminar ${ }^{4,5}$. Freqüentemente, é impossível, na radiografia, distinguir o CBA de outros tumores de pulmão ${ }^{17,18}$.

Em mais do que $70 \%$ dos casos, o diagnóstico foi obtido por biópsia broncoscópica, seguido da biópsia a céu aberto $(26,7 \%)$. Nos estudos publicados na literatura, verifica-se que os diagnósticos são realizados através de avaliação citológica ou anatomopatológica de biópsia percutânea, justificados pela sua localização periférica ${ }^{4}$. $\mathrm{Na}$ série de Merine ${ }^{19}$, por exemplo, $73 \%$ foram 
diagnosticados por biópsia percutânea. No ambulatório de Oncopneumologia da UNIFESP, realiza-se mais biópsia por broncoscopia, devido à sua facilidade em obtê-la.

A maior parte dos pacientes deste estudo, à época do diagnóstico, apresentou doença avançada (60\%), independente do sexo, como ocorre nos outros tipos de carcinoma de células não-pequenas (Tabela 5).

Todos os pacientes com doença localizada foram submetidos à cirurgia $(\mathrm{n}=16)$, sendo que, destes, cinco se submeteram também a algum outro tipo de tratamento complementar (quimioterapia e/ou radioterapia).

Chama a atenção que $1 / 4$ dos pacientes se submeteram apenas a tratamento de suporte, por se negarem a outro tratamento ou por não apresentarem performance status adequado. Nos pacientes com doença localizada, que geralmente se apresenta no estádio I ou II, o tratamento cirúrgico é o de escolha. Já para os pacientes com doença disseminada, a quimioterapia é recomendada devido ao seu potencial de paliação dos sintomas e melhora da qualidade de vida ${ }^{20,21}$.

Entre os exames realizados, o VHS, apesar de ser inespecífico, foi o exame que apresentou a maior porcentagem de pacientes com valores considerados acima do normal $(85,7 \%)$.

No presente trabalho foi avaliado um grande número de pacientes portadores de carcinoma broncogênico, o que nos permitiu estudar as características dos pacientes portadores deste subtipo histológico pequeno que é o carcinoma bronquioloalveolar. Como na literatura não são encontrados dados da população brasileira sobre o carcinoma bronquioloalveolar, mas apenas dados de países desenvolvidos, considera-se que a análise dos pacientes aqui estudados é válida.

Em conclusão, os resultados encontrados são semelhantes aos da literatura mundial, chamando atenção para o fato de que a maior parte dos diagnósticos é realizada através da broncoscopia e em fase avançada.

\section{Potencial Conflito de Interesses:}

Os autores declaram não haver conflito de interesses.

\section{REFERÊNCIAS}

1. Ahmedin JA, Siegal R, Ward E, Murray T, Xu J, Thun MJ. Cancer statistics 2007. CA Cancer J Clin. 2007;57(1):43-66.

2. Instituto Nacional de Câncer (INCA)/ Ministério da Saúde. Estimativas de câncer no Brasil 2005. [acesso em out 2005]. Disponível em: <http://www.inca.gov.br/estimativa/2005>

3. Jamnik S, Tadokoro H, Segreto RA, Costa QJ, Segreto HRC, Yamauti SM, et al. Carcinoma broncogênico: tratamento oncológico clínico e radioterapia. In: Nery LE, Fernandes ALG, Perfeito JA (eds). Guias de medicina ambulatorial e hospitalar UNIFESP / Escola Paulista de Medicina: Pneumologia. São Paulo: Manole; 2006:511-28.

4. Nugué PB, Bevilacqua DJ, Bes FC. Atualização em carcinoma bronquioloalveolar. In: Gomes M, Neder JA, Stelmach R, Leiro LCF (eds). Atualização e reciclagem. Vol VI. São Paulo: Revinter; 2006:193-97.

5. Fernandéz JE, Gómez CC, Corsal GF, Higuera AH, Rodrigues EP. Las diferentes caras del carcinoma bronquioloalveolar. Rev Patol Respir. 2002;5(4):148-52.

6. Levin J. Estatística aplicada a ciências humanas. 2a ed. São Paulo: Harbra; 1987.

7. Read WL, Page NC, Tierney RM, Piccirillo JF, Govendain $R$. The epidemiology of bronchioloalveolar carcinoma over the past two decadas: analysis of the SEER database. Lung Cancer. 2004;45:137-42.

8. Guachalla JU, Czischke CD, Sabbagh EP, Meneses MC, Gajardo GM, Lilayú CR, et al.Carcinoma bronquioloalveolar. Diagnosticos precoz? Experiencia 19941997 en el Instituto Nacional del Torax. Rev Chil Enferm Respir. 2002;18(2):420-24.

9. Hodges CM, Kalemkerian GP. Bronchoalveolar carcinoma masquerading as pneumonia. Prim Care Cancer. 2000;20(7):100-10.

10. Auerbach O, Garfinkel L. The changing pattern of lung adenocarcinoma. Cancer. 1991;68(9):1973-977.

11. Barsky SH, Cameron R, Osann KE, Tometa D, Holmes EC. Rasing incidence of bronchioloalveolar lung carcinoma and its unique clinicopathologic factures. Cancer. 1994;73(4):1163-170.

12. Furak J. The occurrence of bronchioloalveolar lung cancer among our patients. Magy Oncol. 2003;47(4):349-53.

13. Barkley JE, Green MR. Bronchioloalveolar carcinoma. J Clin Oncol. 1996;24:2377-385.

14. Casey K. Neoplastic mimics of pneumonia. Semin Respir Infect. 1995;10(2):231-42.

15. Greco RJ, Stener RM, Goldman S. Bronchioloalveolar cell carcinoma of the lung. Ann Thorac Surg. 1986;41(3):652-56.

16. Dumont P, Gasser B, Rouge C. Bronchioloalveolar carcinoma: Histopatologic study of evolution in a series of 1 a 5 surgically treated patients. Chest. 1998;113(2):391-95.

17. Sider L. Radiographic manifestations of primary bronchogenic carcinoma. Radiol Clin North Am. 1990;28(3):583-96.

18. Travis WD, Garg K, Franklin WA, Wistuba II, Sabloff B, Noguchi M, et al. Evolving concepts in the pathology and computed tomography imaging of lung adenocarcinoma and bronchioloalveolar carcinoma. J Clin Oncol. 2005;23:3279-287.

19. Merine D. Bronchioalveolar carcinoma. Emphasis on localized lesions southern. Med Jur. 1990;82(4):475-80. 
20. Miller VA, Hirsch FR, Johnson PH. Systemic therapy of advanced bronchioloalveolar cell carcinoma: challenges and opportunities. J Clin Oncol. 2005;23:3288-293.

21. Feldman ER, Eagan RT, Schaid DJ. Metastatic bronchioloalveolar carcinoma and metastatic adenocarcinoma of the lung: comparison of clinical manifestations, chemotherapeutic responses and prognosis. Mayo Clin Proc. 1992;67(1):27-32.

\section{Abstract}

Bronchoalveolar carcinoma (BAC) is a subtype of adenocarcinoma, representing less than $5 \%$ of all primary lung tumors. Data are scarce on this type of tumor in Brazil. Objective: The objective was to study the characteristics of patients with BAC treated at the thoracic oncology outpatient department of UNIFESP. Methods: This was a retrospective study of data collected from the patient clinical records at the thoracic oncology outpatient department. Results: Of 1,617 patients with bronchogenic carcinoma, 45 (2.8\%) had a diagnosis of BAC. There was a slight predominance of females (53.5\%), more than $70 \%$ of patients were white, and $64.4 \%$ had a history of smoking. The main symptoms were cough (80\%), expectoration (68.9\%), and dyspnea (60\%). Patients reached our outpatient service after an average of 10.27 months. The predominant radiological images showed a diffuse pattern. The diagnostic method used in most patients was bronchoscopy (71.1\%). Conclusions: Our data are consistent with those in the international literature. Most diagnoses in our outpatient department are obtained by bronchoscopic biopsy.

Key words: Lung cancer, Bronchoalveolar carcinoma, Clinical characteristics 